\title{
INTEGRAL monitoring of 4U 1722-30: the 2008 outburst
}

\section{Antonella Tarana ${ }^{* \dagger}$}

IAPS/INAF, via Fosso del cavaliere 100, 00133 Rome, Italy

E-mail: antonella.tarana@iasf-roma.inaf.it

\section{Angela Bazzano}

IAPS/INAF, via Fosso del cavaliere 100, 00133 Rome, Italy

\section{Pietro Ubertini}

IAPS/INAF, via Fosso del cavaliere 100, 00133 Rome, Italy

\begin{abstract}
As part of a long INTEGRAL monitoring, started in 2003 to discern the soft gamma-ray characteristic of $4 \mathrm{U} 1722-30$, we report here on source results of the recent activity and in particular of the 2008 outburst. From the INTEGRAL and ASM/RXTE light curves, the hardness intensity diagram and spectral analysis using data from JEM-X and IBIS instruments we will show this outburst is different from previous ones. In fact during this two months - long outburst the source was in a hard spectral state characterized only by a different flux rate when compared to the hard spectral state out of the outburst period. Data are well explained with a Componization model with an electron temperature of $28-33 \mathrm{keV}$ and no soft blackbody component is required.
\end{abstract}

The Extreme and Variable High Energy Sky - extremesky2011,

September 19-23, 2011

Chia Laguna (Cagliari), Italy

\footnotetext{
*Speaker.

${ }^{\dagger}$ This work has been supported by the Italian Space Agency through the grant ASI/INAF I/033/10.
} 


\section{Introduction}

$4 \mathrm{U}$ 1722-30, also known as GRS 1724-30, is a bright LMXB located in the Globular Cluster Terzan 2 [4]. The observed Type $1 \mathrm{X}$-ray bursts indicate that the compact object is a weakly magnetized neutron star [4], [5]. The timing properties outlined with RXTE observations suggest that its timing properties are typical of an atoll source [6]. 4U 1722-30 is a persistent though variable source, and it is one of the first neutron star systems from which hard X-ray emission (E $>35 \mathrm{keV}$ ) was detected by SIGMA characterized by a power law spectrum with photon index $\Gamma$ $\sim 1.65$ extending above $100 \mathrm{keV}$ [7], while no emission above $10 \mathrm{keV}$ was reported by EXOSAT [8]. The first broad band observation with BeppoSAX and RXTE allowed to get a detail spectra behavior with a Comptonized spectrum extending up to $200 \mathrm{keV}$, plus an additional soft component (below $3 \mathrm{keV}$ ), described by a blackbody emission [3].

\section{The monitoring of $4 \mathrm{U} 1722-30$ : the 2008 outburst}

The observation period covers from 15 February 2007 to 18 April 2009.

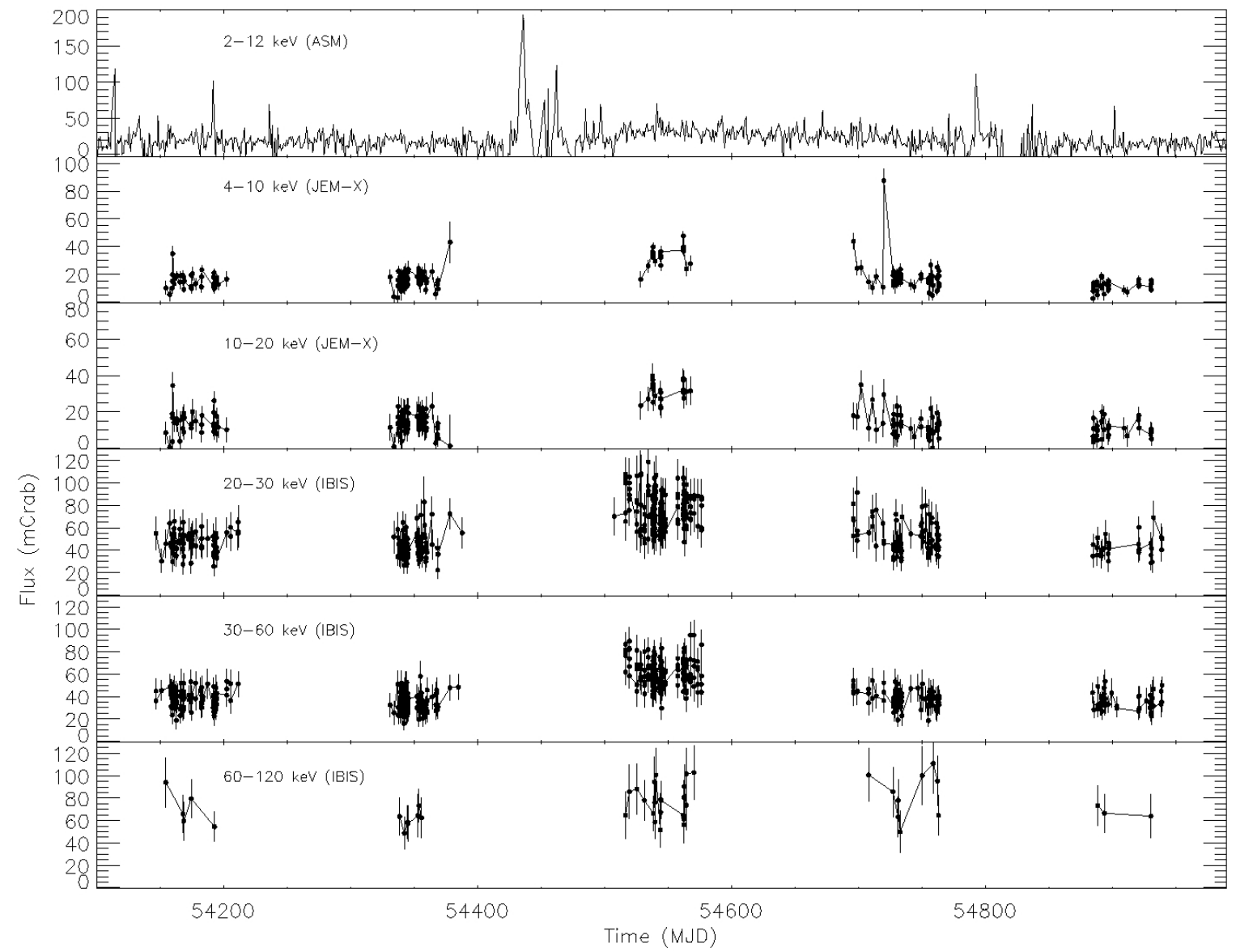

Figure 1: INTEGRAL and ASM/RXTE light curves of 4U 1722-30 during the recent 2008 period outburst.

IBIS observation period (revolution from 530 to 795) is composed by a total of 1840 pointings (science windows, scws) each lasting about 2000 seconds, with the source within 9 degrees from 
the centre of the FOV, while for the spectral analysis only pointings with the source within 4.5 degrees from the centre (FCFOV) have been used yielding to a total of 724 scws. For JEM-X only pointings with the source within 2.4 degrees from the center of the field have been used corresponding to a total of 216 scws. To have a largest and continuous soft X-ray coverage, public data of the ASM/RXTE are also used (from the web site: http://xte.mit.edu/ASM_lc.html).

The INTEGRAL analysis has been performed using OSA 9.1 [2] and by using the hardware capability of the AVES cluster, release 1.4 [9]. For the spectral analysis the XSPEC software v.12.4 [1] has been used. A systematic error of 0.02 has been added to the spectral data. The instrument constant was fixed to 1 for IBIS and keep left for JEM-X.

Figure 1 shows the light curve of the whole observation period with data from ASM/RXTE in the 2-12 keV band, from JEM-X in the 4-10 and 10-20 keV bands and from IBIS in the 20-30, 3060 and 60-120 keV bands, at detection level greater than 3 sigma. From the light curve, we define the "outburst period" the time interval 54420-54730 MJD, the "peak outburst period" the time interval of the rise and peak of the outburst at 54420-54470 MJD, the "after outburst period" the time later than 54730 MJD, the "before the outburst " time before 54420 MJD and as the "outside the outburst" the time before 54420 and after the 54730 MJD.

The outburst started at $54426 \mathrm{MJD}$ and the flux during the "peak period" reached $200 \mathrm{mCrab}$ in the 2-12 keV soft X-ray band while unfortunately there was not INTEGRAL higher energy coverage. During the "outburst period" we detected a high flux level up to high energy bands as evidenced by INTEGRAL coverage (up to $100 \mathrm{mCrab}$ in the 20-30 keV band and $90 \mathrm{mCrab}$ in the 60-120 keV band) for a long period spanning from February 2008 to October 2008. Overall this period, the source was in a hard spectral state as explained in the next section. We note that the source returned to the same mean low flux level in each energy bands during both periods of "after outburst" and "before outburst".
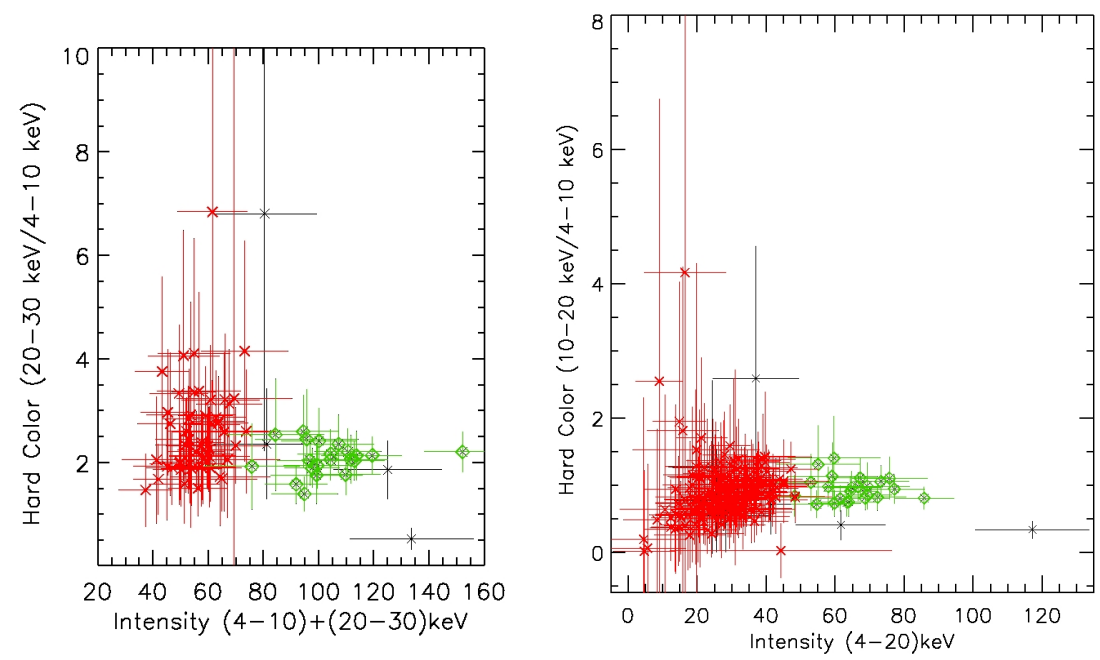

Figure 2: Hardness-intensity diagram with IBIS-JEM-X data (left) and JEM-X only data (right) colorintensity diagram of only last outburst data. The colors identify the speA (red) and speB (green) data sets (see Table 1). 


\section{The hardness intensity diagram and the spectral states evolution}

In Figure 2 it is showed the hardness intensity diagram of the IBIS and JEM-X simultaneous pointings of the whole observation period. The hardness and intensity are respectively defined as the ratio of the rate $(B / A)$ and the sum of the rate $(A+B)$ where $A=4-10 \mathrm{keV}, B=10-20 \mathrm{keV}$ for JEM-X data (Figure 2, right) and $\mathrm{A}=4-10 \mathrm{keV}$ and $\mathrm{B}=20-30 \mathrm{keV}$ for IBIS-JEM-X data (Figure 2, left). Comparing the JEM-X data only with the combined IBIS-JEM-X data no difference is noticed. We note that the source moves in the diagram only changing in flux and not in the hardness value so that it is not expected an evident spectral variation during the observations.

We divided the pointings in the diagram of Figure 2 in two groups for which we extracted the two summed-in spectra. The green data correspond to a high flux spectrum of the "outburst period" of the source while the red data correspond to the low flux spectrum of the "outside outburst period" of the source. The total exposure time is of $111 \mathrm{ks}$ for IBIS and $39 \mathrm{ks}$ for JEM-X for red spectrum and $142 \mathrm{ks}$ for IBIS and $51 \mathrm{ks}$ for JEM-X for the green one.

Table 1: Spectral fitting results for the JEM-X and IBIS spectra of $4 \mathrm{U} 1722-30$. The model is CompTT.

\begin{tabular}{lll}
\hline \hline parameters & speA (red data) & speB (green data) \\
\hline$k T_{0}(\mathrm{keV})^{a}$ & 1.11 & 0.85 \\
$k T_{\mathrm{e}}(\mathrm{keV})$ & $33.00_{-13.28}^{+62.55}$ & $28.53_{-6.79}^{+27.98}$ \\
$\tau$ & $0.71_{-0.58}^{+0.66}$ & $1.08_{-0.63}^{+0.39}$ \\
norm $_{\text {CompTT }}$ & $2.98_{-2.98}^{+1.94} \times 10^{-2}$ & $7.24_{-3.53}^{+2.18} \times 10^{-3}$ \\
\hline$\chi_{r}^{2}$ (d.o.f) & $0.64(42)$ & $1.03(41)$ \\
\hline$F_{4-20 \mathrm{keV}}{ }^{b}$ & $3.76 \times 10^{-10}$ & $8.52 \times 10^{-10}$ \\
$F_{20-200 \mathrm{keV}}$ & $3.62 \times 10^{-10}$ & $8.42 \times 10^{-10}$
\end{tabular}

${ }^{a}$ Fixed parameters

${ }^{b}$ The Fluxes are in units of $\mathrm{erg} \mathrm{s}^{-1} \mathrm{~cm}^{-2}$
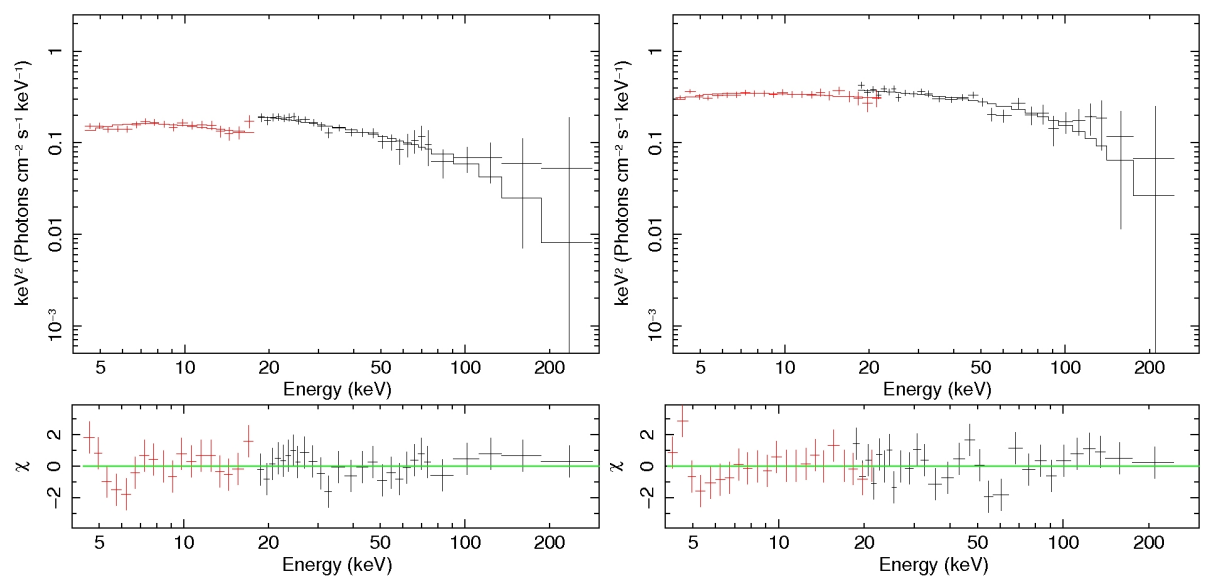

Figure 3: JEM-X and IBIS spectra of 4U 1722-30 during the last observations. Left: red data (speA); Right: green data (speB). 
We try to fit the red and green spectra with different models. In Table 1 spectral fitting results with the Comptonization best fit model [11] are reported. The spectra have slightly different parameters but both correspond to a hard spectral state. The spectral fit does not improve by adding the soft black body component to the Comptonization. In any case it is difficult to constrain the electron temperature of the Comptonization also adding other spectral components. In Table 1 the flux in the band 4-20 keV and 20-200 keV are reported. The two spectra with the model, data and residual are reported in Figure 3.

\section{Comparison of the 2008 outburst behavior with previous ones}

By comparing these results with the previous 2003-2005 INTEGRAL monitoring, reported by Tarana et al. in the 2008 [13] (hereafter T08), we note:

- First of all, the temporal behavior of the outbursts is different. In the left panel of Figure 4 it is showed the light curve for the period 2003-2005 from T08: it is evident that the outbursts in that period were much shorter than the two month-duration in the 2008 one.

- Unfortunately, there was not INTEGRAL full coverage of the 2008 outburst so that it was not possible to follow spectral evolution during the peak and detected the soft and intermediate states. During the previous outbursts was possible to characterize the spectral shape along the evolution from the soft to the hard states (see the right panel of Figure 4 from T08, where the soft state corresponds to the purple data).
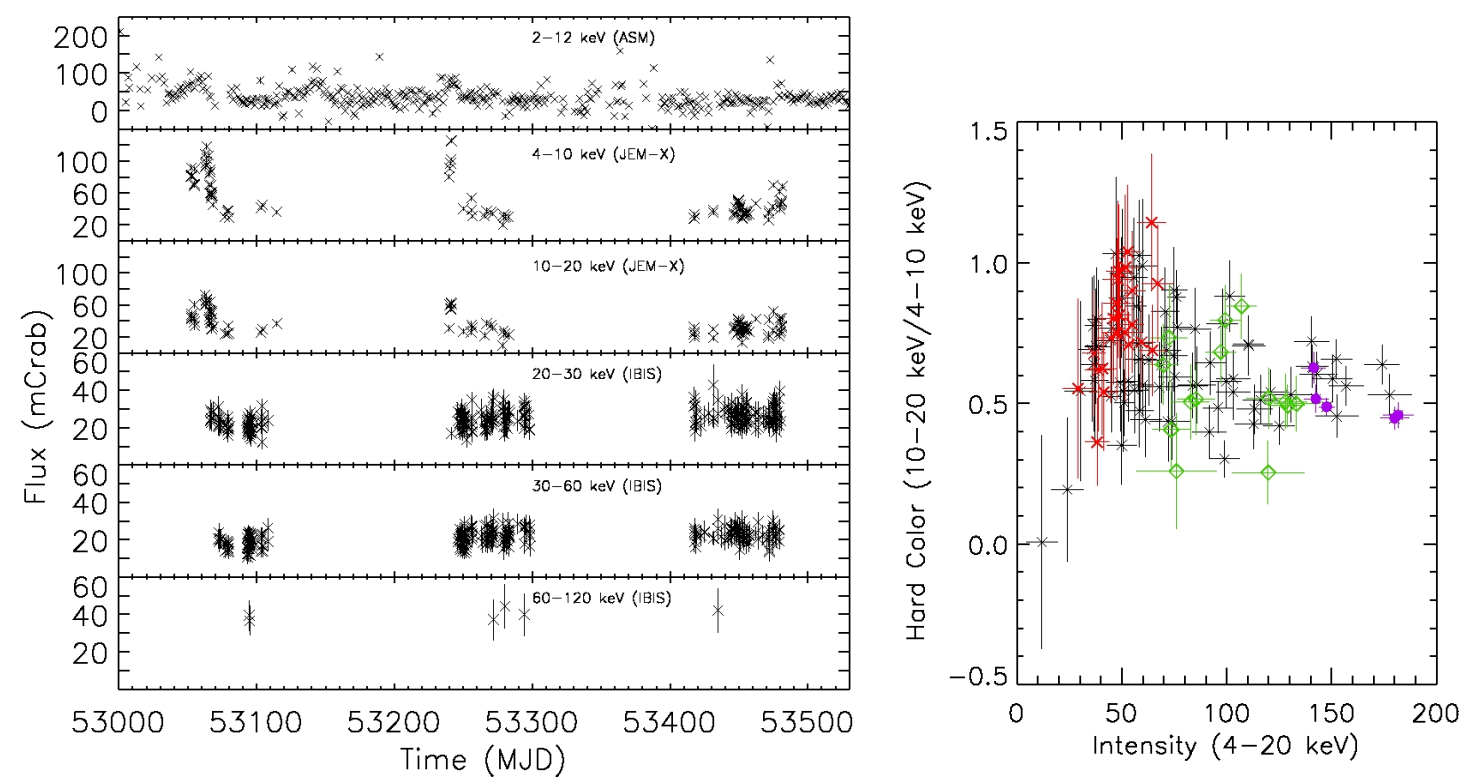

Figure 4: INTEGRAL and ASM/RXTE light curves (left) and hardness diagram (right) of 4U 1722-30 during the 2003-2005 observation period (from [13]).

- Moreover, during the 2008 outburst the source showed hard emission with two flux rate: a high flux hard state which lasted about two month after the peak of the outburst (green spectrum) and a low flux hard state "outside the outburst" (red spectrum). This behavior was not detected during the previous observations (T08), in fact hard X-ray emission, at E $>20 \mathrm{keV}$, was detected after the peak of the outbursts, at a flux level corresponding to the one out of the outburst. 
- Finally, in the 2007-2009 observations the hard spectrum "outside the outburst" (red spectrum) is very similar to the hard spectrum of the previous observation which is reported in Figure 5 (from T08) modeled by a Comptonization with parameters of $k T_{0}$ of $0.8 \mathrm{keV}, k T_{\mathrm{e}}$ of $40 \mathrm{keV}$ and $\tau$ of 0.5 . We note that all the hard states observed in the different outbursts showed, in general, parameters with similar values, and in particular a high temperature of the corona of electron of the order of 30-40 keV which it is ever statistically unconstrained.

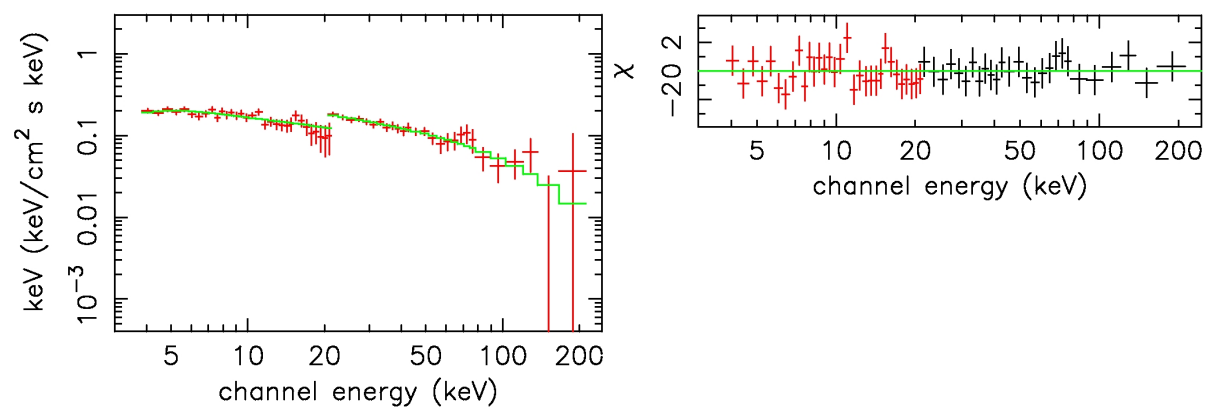

Figure 5: JEM-X and IBIS hard spectrum of the previous 2004-2005 observations (from T08).

All these remarks indicate this source shows outbursts of different time behavior and of different spectral changes. The accretion rate could be the main parameter that drives the spectral variation but we can not exclude other parameters to explain the two hard state of the 2008 outburst. This intriguing behavior suggests to continue the detailed broadband emission study.

\section{References}

[1] Arnauld K. A., in Jacoby G.H., Bames J., eds, ASP Conf. Ser. Vol 101, Astronomical data analysis software and systems V. Astron. Soc. Pa., San Francisco, p.17

[2] Goldwurm A., David P., Foschini L. et al 2003 A\&A, 411, L223

[3] Guainazzi M., et al. A\&A 339, 802, 1998

[4] Grindlay, J. E., Marshall, H. L., Hertz, P., et al. ApJ 240, L21,1980

[5] Swank, J.H., Becker, R. H., Boldt, E. A., et al. 1977, ApJL 212 L73.

[6] Olive, J.F., Barret, D., Boirin, L. et al. 1998 A\&A 333, 942

[7] Barret, D., Olive, J.F., Boirin, L. et al. A\&A 357, L41, 1991

[8] Parmar A.N., Stella, L. \& Giommi, P., A\&A 222, 96, 1989

[9] Federici, M. \& Martino B. PoS(extremesky2009)092, 2009

[10] Mitsuda, K. et al., PASJ, 36, 741, 1984

[11] Titarchuk, L. 1994, ApJ 434, 570

[12] Kuulkers, E., den Hartog, P. R., in’t Zand J. J. M. et al. 2003, A\&A, 399, 663

[13] Tarana A., Bazzano A., Federici M. and Ubertini P., PoS(Integral08)046, 2008, in the paper referred as T08. 